\title{
Neurocognitive and Neuroimaging Predictors of Clinical Outcome in Bipolar Disorder
}

\author{
Carrie E. Bearden • Michelle Woogen • David C. Glahn
}

Published online: 14 September 2010

(C) The Author(s) 2010. This article is published with open access at Springerlink.com

\begin{abstract}
Historically, bipolar disorder has been conceptualized as a disease involving episodic rather than chronic dysfunction. However, increasing evidence indicates that bipolar disorder is associated with substantial inter-episode psychosocial and vocational impairment. Here we review the contributions of neurocognitive deficits and structural and functional neuroanatomic alterations to the observed functional impairments. In particular, compelling evidence now suggests that neurocognitive impairments, particularly in the areas of attention, processing speed, and memory, are associated with functional outcome. Although investigation of the neural correlates of functional disability in bipolar disorder is only in its nascent stages, preliminary evidence suggests that white matter abnormalities may be predictive of poor outcome. A better understanding of the relationship
\end{abstract}

\section{E. Bearden}

Department of Psychiatry and Biobehavioral Sciences, UCLA, 300 Medical Plaza, Suite 2265,

Los Angeles, CA 90095, USA

e-mail: cbearden@mednet.ucla.edu

C. E. Bearden

Department of Psychology, UCLA,

300 Medical Plaza, Suite 2265,

Los Angeles, CA 90095, USA

M. Woogen · D. C. Glahn

Olin Neuropsychiatric Research Center, Institute of Living,

Whitehall Research Building, 200 Retreat Avenue,

Hartford, CT 06106, USA

M. Woogen

e-mail: woogen@hartford.edu

D. C. Glahn $(\bowtie)$

Department of Psychiatry, Yale University School of Medicine,

New Haven, CT, USA

e-mail: david.glahn@yale.edu between neurocognitive and neuroimaging assays and functional outcome has the potential to improve current treatment options and provide targets for new treatment strategies in bipolar disorder.

Keywords Bipolar disorder - Neuropsychology

Neuroanatomy · Functional neuroimaging · Outcome .

Disability

\section{Introduction}

There is growing recognition that bipolar affective disorder is associated with neurocognitive deficits and allied neuroanatomic and neurophysiologic anomalies [1, 2•]. While mood states and psychotropic medication usage influence cognition and brain physiology, observations that many of these neurocognitive and structural and functional neuroanatomic abnormalities predate symptom onset or persist during symptom remission raise the possibility that some changes in brain structure and function are central to the pathophysiology of bipolar affective disorder [3, 4]. Furthermore, accumulating evidence suggests that neurocognitive and neuroimaging factors are associated with clinical outcome, psychosocial functioning, and vocational disability in bipolar disorder [5]. The goal of this review is to synthesize these recent findings to assess their clinical relevance and highlight areas in need of additional research.

Almost two thirds of bipolar patients have some level of inter-episode functional impairment [6,7], and about half are unemployed or have reduced occupational functioning [8]. Thus, although many individuals with bipolar disorder respond well to treatments designed to reduce affective and psychotic symptoms, the ability to achieve functional recovery - the capacity to study, work, engage in recreation, 
live independently, and engage in romantic relationshipsmay be significantly hindered. Therefore, recovery from mood episodes and/or residual symptoms does not necessarily translate into functional recovery. Indeed, many patients with bipolar illness do not regain premorbid levels of psychosocial functioning [9]. Studies of the lifetime course of bipolar disorder consistently find that during periods of symptomatic recovery, bipolar individuals often continue to experience residual mood symptoms that likely contribute to increased levels of disability and decreased psychosocial functioning [10]. Clinical factors-in particular disease chronicity, severity, and subsyndromal depressive symptoms - are associated with poor functional outcome $[5,11]$. In addition, as reviewed in detail below, there is growing evidence for a relationship between neurocognitive function, underlying neuroanatomic abnormalities, and functional outcome across a range of severe neuropsychiatric disorders, including bipolar illness [12-14]. A better understanding of the relationship between neurocognitive and neuroimaging measures and clinical outcome has the potential to improve current treatment options and provide targets for new treatment strategies.

\section{Neurocognitive Findings in Bipolar Disorder}

Although there is clear evidence that patients with bipolar disorder exhibit widespread neurocognitive dysfunction during acute episodes of mania and depression, the discovery that these deficits endure during symptom remission raises the possibility that cognitive impairment may represent a trait rather than a state variable [3]. Euthymic bipolar patients exhibit limitations in several cognitive domains, particularly in measures of executive function, declarative memory, and sustained attention [1, 15-17]. Although euthymic patients often present with residual affective symptoms that may adversely affect performance on cognitive tests [18], even those who have been euthymic for months prior to assessment have marked neuropsychological impairments [19]. Indeed, several recent meta-analyses of neuropsychological performance in euthymic bipolar disorder have documented impairment across a wide variety of cognitive domains [1, 15-17]. In the most recent of these metaanalyses, Kurtz and Gerraty [1] found that nonsymptomatic bipolar patients performed 0.4-0.9 SD below healthy individuals on measures of attention (e.g, Continuous Performance Test; Cohen's $d=0.69$ ), processing speed (e.g, digit symbol substitution; $d=0.66$ ), working memory (e.g, Digit Span Backward; $d=0.65$ ), declarative memory (e.g, Rey or California Verbal Learning Test; $d=0.81$ ), nonverbal declarative memory (e.g, visual reproduction subtest from the Wechsler Memory Scale; $d=0.91$ ), and executive functioning (e.g, Trail Making Test $\mathrm{B} ; d=0.72$ ). Observations of neuropsychological deficits in nonsymptomatic bipolar patients suggest that these impairments may be related to the pathophysiology of the illness and, as described subsequently, are also reasonable predictors of psychosocial functioning and disability [20•].

Evidence that clinically unaffected relatives of patients with bipolar disorder have subtle neurocognitive impairments suggests that such deficits may reflect genetic liability for the illness. A recent meta-analysis reported small but statistically significant differences (e.g, $d<0.5$ ) for unaffected first-degree relatives compared with healthy individuals in the domains of executive functioning and verbal memory [17]. Another recent meta-analysis of 17 published studies included 443 first-degree relatives of bipolar patients and reported cognitive impairments in the range of small to medium effect sizes in the domains of attention (0.08-0.51), verbal learning (0.27-0.33), and executive functioning $(0.22-0.36)$ [21]. Glahn and colleagues [22•] recently reported that three cognitive tests (Digit Symbol Coding, Object Delayed Response Task, and Immediate Facial Memory) are genetically correlated with liability for bipolar disorder, suggesting that the same genetic factors that increase the risk of bipolar disorder influence performance on these tests.

Although many of the neurocognitive impairments found in individuals with bipolar disorder are present during euthymia, it is quite likely that clinical course influences test performance in bipolar disorder. Indeed, age at onset, total number of mood episodes, number of manic episodes, number of depressive episodes, and number and duration of hospitalizations are all factors associated with the degree of neurocognitive impairment among individuals with bipolar disorder [15]. Furthermore, the use of psychotropic medication could impact neurocognitive functioning in bipolar patients. A recent meta-analysis examining the effects of lithium on cognitive performance in bipolar disorder in 12 studies involving 276 lithium-treated and 263 lithium-free patients found a small but significant impairment in lithium-treated patients in immediate verbal learning and memory (effect size, 0.24 ) and creativity (effect size, 0.33 ) [5]. In contrast, no significant impairments were found for delayed verbal memory, visual memory, attention, executive functioning, processing speed, or psychomotor performance. Some antidepressant medications have been shown to yield adverse cognitive effects, particularly those with anticholinergic properties [23]. However, other studies have failed to find significant effects of such medications on cognition [24, 25]. Although few studies have examined neurocognitive performance in unmedicated bipolar patients, Goswami and colleagues [26] found no significant differences in neurocognitive test performance between 22 drug-free euthymic bipolar patients and 22 medicated euthymic patients. Together, these data suggest that although clinical course 
and medication usage may influence cognitive performance in bipolar disorder, these effects seem to explain only a fraction of the observed impairments.

Growing evidence indicates that the neurocognitive impairments contribute to poorer psychosocial functioning and increased functional disability in bipolar disorder [12-14, $20 \bullet, 27,28]$. For example, executive dysfunction at initial assessment has been linked to lower levels of functional recovery in this population both cross-sectionally and longitudinally [13, 14, 28]. Bonnin and colleagues [29•] recently found that subthreshold depressive symptoms and working memory function (digits backward) were specific predictors of occupational functioning 4 years later. Similarly, bipolar patients with declarative memory deficits are less likely to return to premorbid psychosocial or occupational functioning $[11,14,28,29 \bullet]$.

Schizophrenia research has recently begun to distinguish between functional capacity (i.e, an estimate of one's ability to perform tasks relevant to everyday functioning) and actual performance (i.e, what one actually does in everyday settings). This distinction is important, as performance can be influenced by functional capacity as well as environmental, motivational, and other factors. Bowie and colleagues [20 ${ }^{\bullet}$ recently performed a series of confirmatory path analyses to determine how neurocognitive deficit influences real world functioning and disability in bipolar disorder. A total of 130 community-dwelling individuals with bipolar disorder were assessed with neuropsychological tests, symptom measures, and performance-based social and adaptive functional competence measures in three domains of real world functioning (community and household activities, work skills, and interpersonal relationships). In all models, the relationship between neurocognition and outcome was largely mediated by competence. Neurocognition was related to activities directly and through a mediated relationship with adaptive competence. In contrast, work skills were entirely mediated by adaptive and social competence. The relationship between neurocognition and interpersonal relationships was mediated by social competence. These findings imply that functional disability may persist in patients with bipolar disorder even after symptomatic recovery due to neurocognitive and skill deficits. Conversely, even if a patient acquires certain neurocognitive skills but continues to experience mild mood symptomatology, changes in real world behavior might lag or not manifest at all.

In summary, significant evidence suggests that many individuals with bipolar disorder have at least some degree of neurocognitive deficit. Although these deficits may be influenced by clinical course/severity or psychotropic medication usage, they also seem to be associated with the genetic liability for the illness, suggesting that neurocognitive deficits are an important aspect of the presentation of bipolar disorder.
Finally, neurocognitive impairments impact real world functioning of patients with bipolar disorder.

\section{Neuroanatomic Findings in Bipolar Disorder}

The most consistently documented neuroanatomic abnormalities in adult patients with bipolar disorder are lateral ventricle enlargement $(+17 \%)$ and increased rates of deep white matter hyperintensities (Odds Ratio, 2.49) [2•]. Reduced area or volume of the corpus callosum is also a robust finding across studies [30]. Additional evidence for white matter involvement in bipolar disorder comes from studies finding alterations in white matter tract organization $[31,32]$ and regional white matter volume reductions [33]. In addition, some recent evidence indicates that white matter abnormalities may be stable, trait-based abnormalities that reflect genetic liability to the illness [31, 34]. Kieseppa and colleagues [33] found decreased left hemisphere white matter volume in bipolar probands and their nonbipolar co-twins. Similarly, McDonald and colleagues [34] found that the genetic risk of bipolar disorder was associated with white matter reduction in the left frontal and temporoparietal regions, suggesting that left frontotemporal disconnectivity may be a genetically controlled neuroanatomic abnormality associated with bipolar illness. Finally, in a large Dutch twin sample, van der Schot and colleagues [35•] found that reduction in overall white matter volume was related to the genetic risk of bipolar disorder, whereas significant environmental correlations were observed for cortical gray matter.

In general, findings of gray matter alterations in bipolar disorder are more variable across studies, which is likely at least partially attributable to the now well-documented effects of lithium on gray matter volume $[2 \cdot 36]$. Although a recent meta-analysis revealed an effect size of 1.17 for reduced volume of the left anterior cingulate in bipolar patients relative to controls, this finding was not significant across studies due to the high between-study heterogeneity [2•]. Kempton and colleagues [37], using voxel-based morphometry in patients with bipolar I disorder, their relatives with major depression, healthy relatives, and controls, found group differences in the left insula, cerebellum, and substantia nigra; increased left insula volume in particular was associated with genetic preposition to bipolar disorder independent of clinical phenotype. In contrast, increased left substantia nigra volume was specific to those with the clinical phenotype of bipolar I disorder. Changes uniquely associated with the absence of a clinical diagnosis in bipolar relatives were observed in the left cerebellum, suggesting that there may be dissociable genetic and phenotypic influences on brain structure in bipolar disorder.

Given the substantial body of literature on neuroanatomic changes in bipolar disorder [2•], surprisingly few 
studies have examined their relationship to outcome. In patients with schizophrenia and those at risk of the illness, this relationship has been examined in several studies. For example, accelerated ventricular enlargement in the 3 years following illness onset was associated with poor outcome in first-episode schizophrenia, whereas a progressive decrement in frontal lobe white matter was associated with greater negative symptom severity [38]. More recently Karlsgodt and colleagues [39] found that the structural integrity of medial temporal white matter tracts was predictive of functional outcome in adolescents at high risk of developing psychosis. Given the accumulating evidence that disrupted white matter integrity may be central to the pathophysiology of bipolar disorder, white matter abnormalities represent a promising candidate for a neuroanatomic predictor of outcome.

Two prior studies examined white matter hyperintensities as indicators of treatment resistance [40] and poor outcome [41] in bipolar disorder. Moore and colleagues [41] categorized patients with bipolar disorder as good or poor outcome based on treatment response and level of functioning, finding that poor outcome group members had significantly more deep and more severe subcortical punctate white matter hyperintensities relative to those in the good outcome group and healthy controls. Regenold and colleagues [40] found that an index of treatment resistance correlated significantly with deep white matter hyperintensity volume, as well as measures of abnormal brain glucose metabolism (sorbitol and fructose) in bipolar patients but not in other patients (i.e, those with schizophrenia and neurologic controls).

In summary, there is strong evidence to date implicating neurocognitive factors in bipolar patients as key determinants of functional outcome. Given that these cognitive abnormalities likely are reflective of underlying abnormalities in brain structure and function, we propose that microstructural white matter alterations may contribute to poor outcome.

\section{Functional Neuroimaging Findings in Bipolar Disorder}

Similar to the literature relating abnormalities of brain structure to poor outcome in schizophrenia, recent functional MRI studies also have begun to document such a relationship. In particular, Fusar-Poli and colleagues [42] found that in youth with prodromal signs of psychosis, clinical and functional improvement over follow-up was associated with a longitudinal increase in activation in the anterior cingulate and right parahippocampal gyrus during performance on an N-back task. Similarly, in individuals at high clinical risk of psychosis, Sabb and colleagues [43] found that baseline neural activity in the left inferior frontal gyrus during performance on a language processing task was predictive of severity of positive formal thought disorder and poor social outcome at follow-up.

To date, no such studies have been conducted in patients with bipolar disorder. However, emerging literature suggests that the neurophysiology of bipolar disorder involves frontal hypoactivation with concomitant disinhibition (i.e, relative hyperactivation) of limbic structures [44, 45]. The subgenual prefrontal cortex modulates the affective output of limbic and paralimbic structures and cognitive output from the prefrontal cortex. Recent evidence from functional MRI studies using affective processing paradigms (ie, reacting to stimuli with positive or negative emotional valence) suggests that patients with bipolar disorder may disproportionately engage limbic structures during emotionally valent tasks, regardless of mood state [46, 47], although the direction of effects has not been consistent across studies. Taken together, these studies suggest that corticolimbic dysregulation may underlie the emotional dysregulation and cognitive impairments associated with bipolar disorder [45, 48]. Moreover, exaggerated medial prefrontal cortical and subcortical (putamen and amygdala) responses to emotional signals have been observed in bipolar I patients and their nonbipolar relatives, suggesting that such responses may represent heritable neurobiological abnormalities underlying bipolar disorder [49].

\section{Implications for Treatment}

Given that similar cognitive impairments and associated psychosocial and functional disabilities are seen in patients with bipolar disorder and those with schizophrenia, the adaptation of treatment strategies that have proven beneficial for schizophrenia patients may also be efficacious for bipolar patients. In particular, cognitive remediation has been associated with significant, although modest, improvements in cognitive performance and psychosocial functioning in schizophrenia patients [50]. However, treatment of cognitive impairment in bipolar illness remains largely unexplored. The development of treatments that target cognitive impairments and functional status is an important area of future investigation in bipolar disorder. Two small studies have found that rehabilitative interventions such as cognitive remediation and supported employment may be effective in improving vocational outcomes for bipolar patients, but this will require replication in larger investigations [9].

\section{Conclusions}

Although bipolar disorder historically has been viewed as a disease involving only episodic dysfunction, increasing evidence indicates that this is not the case for a substantial 
proportion of patients. Collectively, the data reviewed above provide compelling evidence that cognitive impairments are present across multiple domains - particularly in the areas of attention, processing speed, and memory - in most bipolar patients. These difficulties are observable at or soon after illness onset, and persist throughout the course of illness. Given that clinically unaffected relatives of patients with bipolar disorder have similar but milder neurocognitive impairments, such deficits may reflect genetic liability for the illness.

Importantly, these cognitive deficits appear to have implications for real world functioning. Although investigation of the neural correlates of functional disability in bipolar disorder is only in its nascent stages, given strong commonalities with schizophrenia, it is anticipated that similar relationships between structural and functional neuroanatomic abnormalities and outcome in bipolar patients will be identified. The rich literature in schizophrenia - and in individuals at risk of the illness - could serve to inform areas of future research needed in bipolar disorder.

As recommended by Harvey and colleagues [9], approaches used in schizophrenia research - in particular, longitudinal assessment of cognition, neurophysiology, and psychosocial function across variations in clinical state, and separate assessment of functional capacity and real world functioning - will be highly informative when applied to the study of patients with bipolar illness. A clear need exists for objective methods of assessing real world functional abilities, as subjective self-assessments are likely to be influenced by current symptoms or other illness features [9]. Thus far, few studies have investigated specific aspects of functional status in bipolar patients and their associations with neuroanatomic, clinical, and treatment-related factors. Such investigations are critical for understanding the array of determinants of disability in bipolar illness.

Disclosure No potential conflicts of interest relevant to this article were reported.

Open Access This article is distributed under the terms of the Creative Commons Attribution Noncommercial License which permits any noncommercial use, distribution, and reproduction in any medium, provided the original author(s) and source are credited.

\section{References}

Papers of particular interest, published recently, have been highlighted as:

\section{- Of importance}

1. Kurtz M, Gerraty R: A meta-analytic investigation of neurocognitive deficits in bipolar illness: profile and effects of clinical state. Neuropsychology 2009, 23:551-562.
2. - Kempton M, Geddes J, Ettinger U, et al.: Meta-analysis, database, and meta-regression of 98 structural imaging studies in bipolar disorder. Arch Gen Psychiatry 2008, 65:1017-1032. This influential meta-analysis reviewed the existent evidence for neuroanatomic abnormalities in bipolar disorder, reporting lateral ventricle enlargement and increased rates of deep white matter hyperintensities in the illness. In addition, lithium use was associated with increased gray matter volume in patients.

3. Glahn D, Therman S, Manninen M, et al.: Spatial working memory as an endophenotype for schizophrenia. Biol Psychiatry 2003, 53:624-626.

4. Bearden CE, Hoffman KM, Cannon TD: The neuropsychology and neuroanatomy of bipolar affective disorder: a critical review. Bipolar Disord 2001, 3:106-150; discussion 151-153.

5. Wingo A, Wingo T, Harvey P, Baldessarini R: Effects of lithium on cognitive performance: a meta-analysis. J Clin Psychiatry 2009, 70:1588-1597.

6. Tohen M, Zarate CA Jr, Hennen J, et al.: The McLean-Harvard First-Episode Mania study: prediction of recovery and first recurrence. Am J Psychiatry 2003, 160:2099-2107.

7. Suppes T, Leverich GS, Keck PE, et al.: The Stanley Foundation Bipolar Treatment Outcome Network. II. Demographics and illness characteristics of the first 261 patients. J Affect Disord 2001, 67:45-59.

8. Altshuler L, Tekell J, Biswas K, et al.: Executive function and employment status among veterans with bipolar disorder. Psychiatr Serv 2007, 58:1441-1447.

9. Harvey P, Wingo A, Burdick K, Baldessarini R: Cognition and disability in bipolar disorder: lessons from schizophrenia research. Bipolar Disord 2010, 12:364-375.

10. Judd L, Akiskal H, Schettler P, et al.: Psychosocial disability in the course of bipolar I and II disorders: a prospective, comparative, longitudinal study. Arch Gen Psychiatry 2005, 62:1322-1330.

11. Martinez-Aran A, Vieta E, Torrent C, et al.: Functional outcome in bipolar disorder: the role of clinical and cognitive factors. Bipolar Disord 2007, 9:103-113.

12. Martino D, Marengo E, Igoa A, et al.: Neurocognitive and symptomatic predictors of functional outcome in bipolar disorders: a prospective 1 year follow-up study. J Affect Disord 2009, 116:37-42.

13. Jaeger J, Berns S, Loftus $S$, et al.: Neurocognitive test performance predicts functional recovery from acute exacerbation leading to hospitalization in bipolar disorder. Bipolar Disord 2007, 9:93-102.

14. Martinez-Aran A, Vieta E, Colom F, et al.: Cognitive impairment in euthymic bipolar patients: implications for clinical and functional outcome. Bipolar Disord 2004, 6:224-232.

15. Robinson L, Thompson J, Gallagher P, et al.: A meta-analysis of cognitive deficits in euthymic patients with bipolar disorder. $\mathrm{J}$ Affect Disord 2006, 93:105-115.

16. Torres I, Boudreau V, Yatham L: Neuropsychological functioning in euthymic bipolar disorder: a meta-analysis. Acta Psychiatr Scand Suppl 2007, 434:17-26.

17. Arts B, Jabben N, Krabbendam L, van Os J: Meta-analyses of cognitive functioning in euthymic bipolar patients and their firstdegree relatives. Psychol Med 2008, 38:771-785.

18. Ferrier IN, Stanton BR, Kelly TP, Scott J: Neuropsychological function in euthymic patients with bipolar disorder. $\mathrm{Br} \mathrm{J}$ Psychiatry 1999, 175:246-251.

19. Thompson J, Gallagher P, Hughes J, et al.: Neurocognitive impairment in euthymic patients with bipolar affective disorder. Br J Psychiatry 2005, 186:32-40.

20. - Bowie C, Depp C, McGrath JA, et al.: Prediction of real-world functional disability in chronic mental disorders: a comparison of schizophrenia and bipolar disorder. Am J Psychiatry 2010 May 17 (Epub ahead of print). This landmark study is the first large-scale 
study of the impact of neurocognition on real world functioning and disability in bipolar disorder and schizophrenia. Although there was greater disability in schizophrenia, neurocognition predicted worse functioning in all outcome domains in both disorders, suggesting a role for neurocognition in the disability of both illnesses.

21. Bora E, Yucel M, Pantelis C: Cognitive endophenotypes of bipolar disorder: a meta-analysis of neuropsychological deficits in euthymic patients and their first-degree relatives. J Affect Disord 2009, 113:1-20.

22. - Glahn D, Almasy L, Barguil M, et al.: Neurocognitive endophenotypes for bipolar disorder identified in multiplex multigenerational families. Arch Gen Psychiatry 2010, 67:168177. We examined cognitive functioning in a large sample of bipolar pedigrees and found three cognitive measures that are genetically correlated with the illness. These findings suggest that neurocognitive measures are strong candidates for endophenotypes for bipolar disorder, as common genetic factors influence risk of the disorder and neuropsychological performance.

23. Amado-Boccara I, Gougoulis N, Poirier Littré M, et al.: Effects of antidepressants on cognitive functions: a review. Neurosci Biobehav Rev 1995, 19:479-493.

24. Senturk V, Goker C, Bilgic A, et al.: Impaired verbal memory and otherwise spared cognition in remitted bipolar patients on monotherapy with lithium or valproate. Bipolar Disord 2007, 9 (Suppl 1):136-144.

25. Tonnby B, Nilsson H, Aldenkamp A, et al.: Withdrawal of antiepileptic medication in children. Correlation of cognitive function and plasma concentration - the multicentre 'Holmfrid' study. Epilepsy Res 1994, 19:141-152.

26. Goswami U, Sharma A, Varma A, et al.: The neurocognitive performance of drug-free and medicated euthymic bipolar patients do not differ. Acta Psychiatr Scand 2009, 120:456-463.

27. Atre-Vaidya N, Taylor MA, Seidenberg M, et al.: Cognitive deficits, psychopathology, and psychosocial functioning in bipolar mood disorder. Neuropsychiatry Neuropsychol Behav Neurol 1998, 11:120-126.

28. Bora E, Vahip S, Akdeniz F, et al.: The effect of previous psychotic mood episodes on cognitive impairment in euthymic bipolar patients. Bipolar Disord 2007, 9:468-477.

29. - Bonnín C, Martínez-Arán A, Torrent C, et al.: Clinical and neurocognitive predictors of functional outcome in bipolar euthymic patients: a long-term, follow-up study. J Affect Disord 2010, 121:156-160. This study demonstrated that neuropsychological indices and depression measures predicted psychosocial and occupational functioning 4 years after assessment in a sample of 32 bipolar patients. The results highlight the stability of the cognitive markers for prediction of clinical outcomes.

30. Arnone D, McIntosh A, Chandra P, Ebmeier K : Meta-analysis of magnetic resonance imaging studies of the corpus callosum in bipolar disorder. Acta Psychiatr Scand 2008, 118:357-362.

31. Chaddock C, Barker G, Marshall N, et al.: White matter microstructural impairments and genetic liability to familial bipolar I disorder. Br J Psychiatry 2009, 194:527-534.

32. Wang F, Jackowski M, Kalmar J, et al.: Abnormal anterior cingulum integrity in bipolar disorder determined through diffusion tensor imaging. Br J Psychiatry 2008, 193:126-129.

33. Kieseppa T, van Erp TG, Haukka J, et al.: Reduced left hemispheric white matter volume in twins with bipolar I disorder. Biol Psychiatry 2003, 54:896-905.
34. McDonald C, Bullmore ET, Sham PC, et al.: Association of genetic risks for schizophrenia and bipolar disorder with specific and generic brain structural endophenotypes. Arch Gen Psychiatry 2004, 61:974-984.

35. • van der Schot A, Vonk R, Brans R, et al.: Influence of genes and environment on brain volumes in twin pairs concordant and discordant for bipolar disorder. Arch Gen Psychiatry 2009, 66:142-151. This study showed that decreased white matter was related to the genetic risk of bipolar disorder in 50 twin pairs discordant for bipolar disorder and 67 control twin pairs. The data provide additional support for the notion that neuroanatomic abnormalities observed in bipolar disorder may be influenced by the same genetic factors that increase liability for the illness.

36. Bearden C, Thompson P, Dalwani M, et al.: Greater cortical gray matter density in lithium-treated patients with bipolar disorder. Biol Psychiatry 2007, 62:7-16.

37. Kempton M, Haldane M, Jogia J, et al.: Dissociable brain structural changes associated with predisposition, resilience, and disease expression in bipolar disorder. J Neurosci 2009, 29:10863-10868.

38. Ho B, Andreasen N, Nopoulos P, et al.: Progressive structural brain abnormalities and their relationship to clinical outcome: a longitudinal magnetic resonance imaging study early in schizophrenia. Arch Gen Psychiatry 2003, 60:585-594.

39. Karlsgodt K, Niendam T, Bearden C, Cannon T: White matter integrity and prediction of social and role functioning in subjects at ultra-high risk for psychosis. Biol Psychiatry 2009, 66:562-569.

40. Regenold W, Hisley K, Phatak P, et al.: Relationship of cerebrospinal fluid glucose metabolites to MRI deep white matter hyperintensities and treatment resistance in bipolar disorder patients. Bipolar Disord 2008, 10:753-764.

41. Moore P, Shepherd D, Eccleston D, et al.: Cerebral white matter lesions in bipolar affective disorder: relationship to outcome. $\mathrm{Br} \mathrm{J}$ Psychiatry 2001, 178:172-176.

42. Fusar-Poli P, Broome M, Matthiasson P, et al.: Spatial working memory in individuals at high risk for psychosis: longitudinal fMRI study. Schizophr Res 2010 Aug 3 (Epub ahead of print).

43. Sabb F, van Erp T, Hardt M, et al.: Language network dysfunction as a predictor of outcome in youth at clinical high risk for psychosis. Schizophr Res 2010, 116:173-183.

44. Brooks JO 3 rd, Wang PW, Bonner JC, et al.: Decreased prefrontal, anterior cingulate, insula, and ventral striatal metabolism in medication-free depressed outpatients with bipolar disorder. J Psychiatr Res 2009, 43:181-188.

45. Strakowski S, Delbello M, Adler C: The functional neuroanatomy of bipolar disorder: a review of neuroimaging findings. Mol Psychiatry 2005, 10:105-116.

46. Malhi GS, Lagopoulos J, Sachdev P, et al.: Cognitive generation of affect in hypomania: an fMRI study. Bipolar Disord 2004, 6:271-285.

47. Robinson J, Bearden C, Monkul E, et al.: Fronto-temporal dysregulation in remitted bipolar patients: an fMRI delayed-non-match-tosample (DNMS) study. Bipolar Disord 2009, 11:351-360.

48. Drevets W, Price J, Furey M: Brain structural and functional abnormalities in mood disorders: implications for neurocircuitry models of depression. Brain Struct Funct 2008, 213:93-118.

49. Surguladze S, Marshall N, Schulze K, et al.: Exaggerated neural response to emotional faces in patients with bipolar disorder and their first-degree relatives. Neuroimage 2010, 53:58-64.

50. McGurk S, Twamley E, Sitzer D, et al.: A meta-analysis of cognitive remediation in schizophrenia. Am J Psychiatry 2007, 164:1791-1802 